\title{
R WAVES INTERRUPTING T WAVES
}

\author{
BY \\ F. H. SMIRK \\ From the Department of Medicine, University of Otago, New Zealand \\ Received July 25, 1948
}

In the course of some experiments on anæsthetized dogs and cats concerned with the production experimentally of ventricular flutter and ventricular fibrillation, Fastier and Smirk (1948) noted that R waves sometimes appeared on the descent of the antecedent $T$ waves, shortly before the onset of venttricular flutter. The flutter was induced by a substance amarin * which had been found by one of us (F.H.S.) to alter the response of the animals to adrenaline so that a moderate dose of adrenaline, ordinarily well tolerated, would now give rise to ventricular flutter.

These observations led me to seek more closely for such phenomena in human electrocardiograms. The present paper concerns 17 patients in whom $R$ waves have been observed on the $T$ waves of antecedent complexes. It seems that interruptions of $T$ waves by $R$ waves are not very uncommon, are likely to prove important prognostically, and seem to provide an indication for treatment.

Little has been written on this subject either from the experimental or from the clinical standpoint, and such information as is available may be unfamiliar to some of the authors who have published records without commenting upon the phenomenon. The first reference I was able to discover to the occurrence of an $R$ wave on a $T$ wave was in a footnote by Katz (1928) in the course of his comprehensive review on the $T$ wave. Katz mentioned here that an example of an $R$ wave on a $T$ wave has been observed by Dr. Ashman, three examples by Drs. Feil and Seigel, and three by himself. He mentioned that the only published record was by Wenkebach and Winterberg (1927) and that Wiggers had seen it in dogs. I have been unable to find any record of subsequent reports on the finding of $R$ waves on the $T$ waves with the exception of Scherf

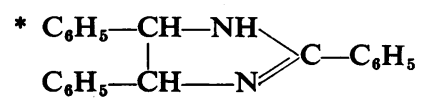

and Boyd (1940) and of Ashman and Hull (1941) in their books on electrocardiography; they confine themselves to the statement that an $R$ wave may encroach on the descent of the $T$ wave.

The published electrocardiograms show many examples in which $\mathbf{R}$ waves appear to arise on the descent of $T$ waves but, with the exception referred to above, I have found so far no corresponding comment by the authors.

Theoretically $\mathbf{R}$ waves might occur superimposed upon $\mathrm{T}$ waves in several ways. Most of these theoretical possibilities have been given practical illustration in the 17 cases here reported.

(1) A ventricular complex of supraventricular origin could be interrupted by another ventricular complex of supraventricular origin (Cases 3, 4, 13, 14, 15, 16; Fig. 3 and 9). This might occur in auricular paroxysmal tachycardia in auricular fibrillation (Fig. 3 and 9), in auricular flutter, and possibly with very premature auricular systoles.

(2) A ventricular complex of supraventricular origin could be interrupted by a premature ventricular complex (Cases 1, 4, 5, 6, 7, 8, 9, 10, 11, 12, 15, 17; Fig. 6 and 7).

(3) A premature ventricular complex could be interrupted by a ventricular complex of supraventricular origin (Case 4; Fig. 4).

(4) A premature ventricular complex could be interrupted by another premature ventricular complex (Cases 1, 2, 4, 5, 7, 8; Fig. 1, 2, 4, 5, and 8). This last might occur with single pairs of ventricular premature beats (Fig. 1) or in the course of ventricular paroxysmal tachycardia (Fig. 2).

Reference to published cardiographic tracings illustrating a variety of cardiological conditions suggests that some of the other theoretical possibilities may have been realized. For example interruptions of paroxysmal auricular tachycardia (Graybiel and White, 1946) in which the $P$ waves appeared in the S-T segments. 


\section{Case Reports}

Case 1, male, aged 59 years. Retrosternal pain twelve hours before admission. The electrocardiographic diagnosis was that of anterior apical infarction. In two instances in abot 6 seconts of electrocardiographic recording examples were found of an ectopic ventricular complex interrupted before its completion by a subsequent ectopic complex (Fig. 1). Both interruptions occurred on the latter half of the $T$ wave.

The patient was improving during the first 19 days and then had an extension of the cardiac infarction with a further fall in the blood pressure. Next day he was noticed to be breathing ster- torously. Shortly after he tried to sit up and died suddenly, as was thought, from ventricular flutter. The autopsy revealed multiple small infarcted areas in the anterior apical region.

Case 2, male, aged 46 years. Cardiac infarction. Severe retro-sternal pain of 36 hours duration. About one month after admission the patient developed a ventricular paroxysmal tachycardia and was treated by quinidine which restored a natural regular rhythm. The patient was discharged in good condition.

The electrocardiograms were indefinite in regard to the clinical diagnosis of cardiac infarction. The terminal deflection of the first run of tachycardia
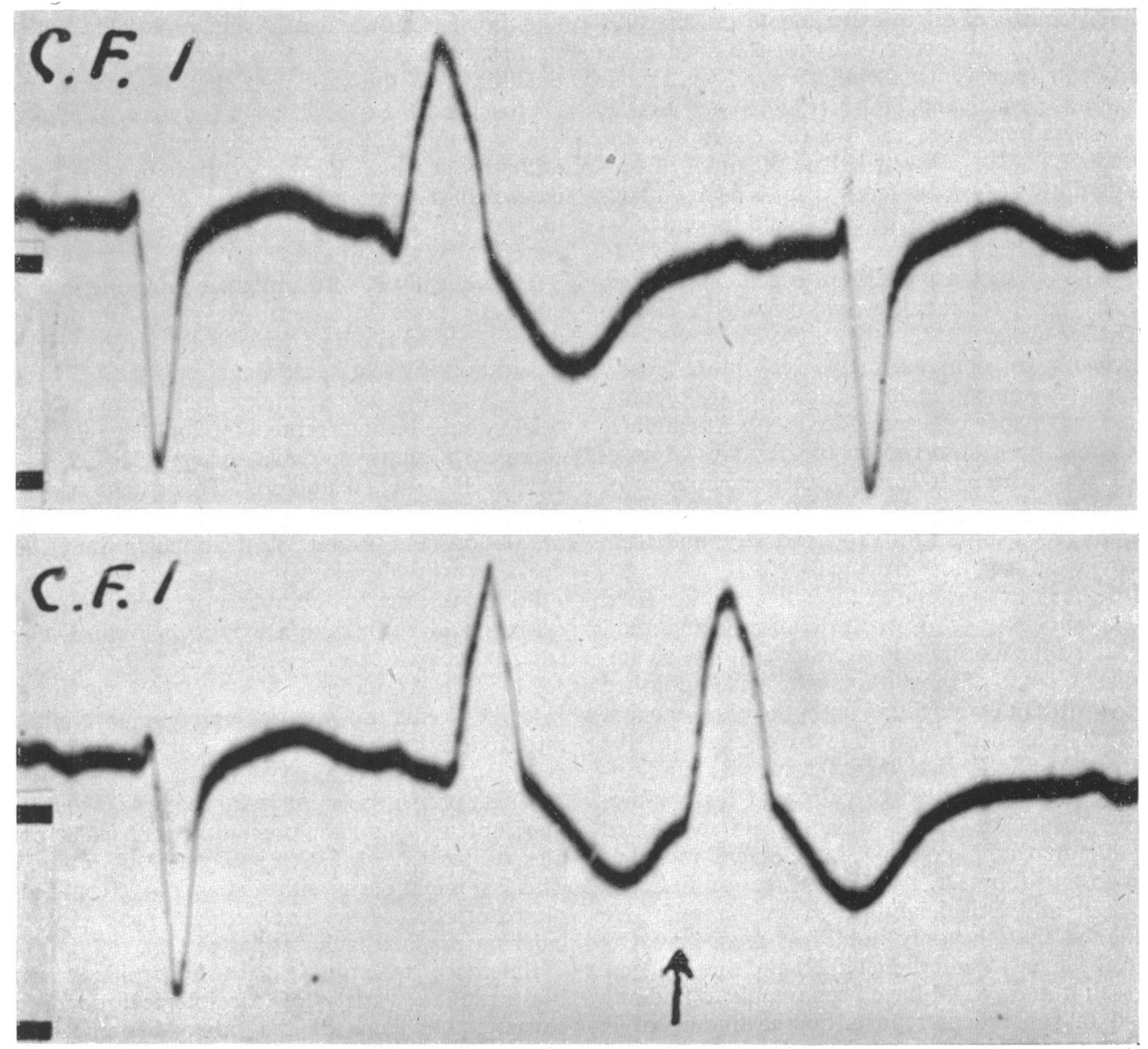

Fig. 1.-Case 1. The upper trace shows a single uninterrupted premature ventricular systole and the lower shows a pair of premature ventricular systoles of which the second interrupts the terminal deflection of the first. The interrupted complex may be compared in shape with the complete complex in the upper trace and with the complex that follows it. Time intervals $0.1 \mathrm{sec}$. 
in lead III and the ventricular complex towards the In the middle of the paroxysm the individual comend of the lead III strip indicate the shape and duration of uninterrupted ventricular complexes (Fig. 2). plexes differ in duration and this seems to depend chiefly on the point at which the T-like terminal

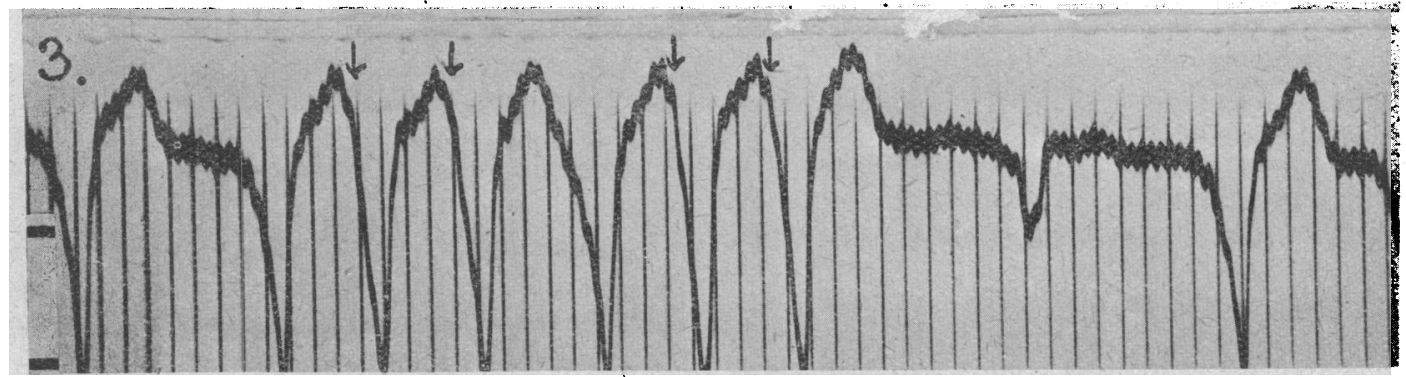

Fio. 2.-Case 2. The trace shows a paroxysm of ventricular tachycardia. This complex at the end of the paroxysm and the complexes at the extreme left and right of the trace are uninterrupted. The arrows mark the points where complexes are interrupted during the paroxysm. Comparison with intact complexes indicates how much of the interrupted complexes have been cut off by the beginnings of new ventricular complexes. Time intervals $0.05 \mathrm{sec}$.
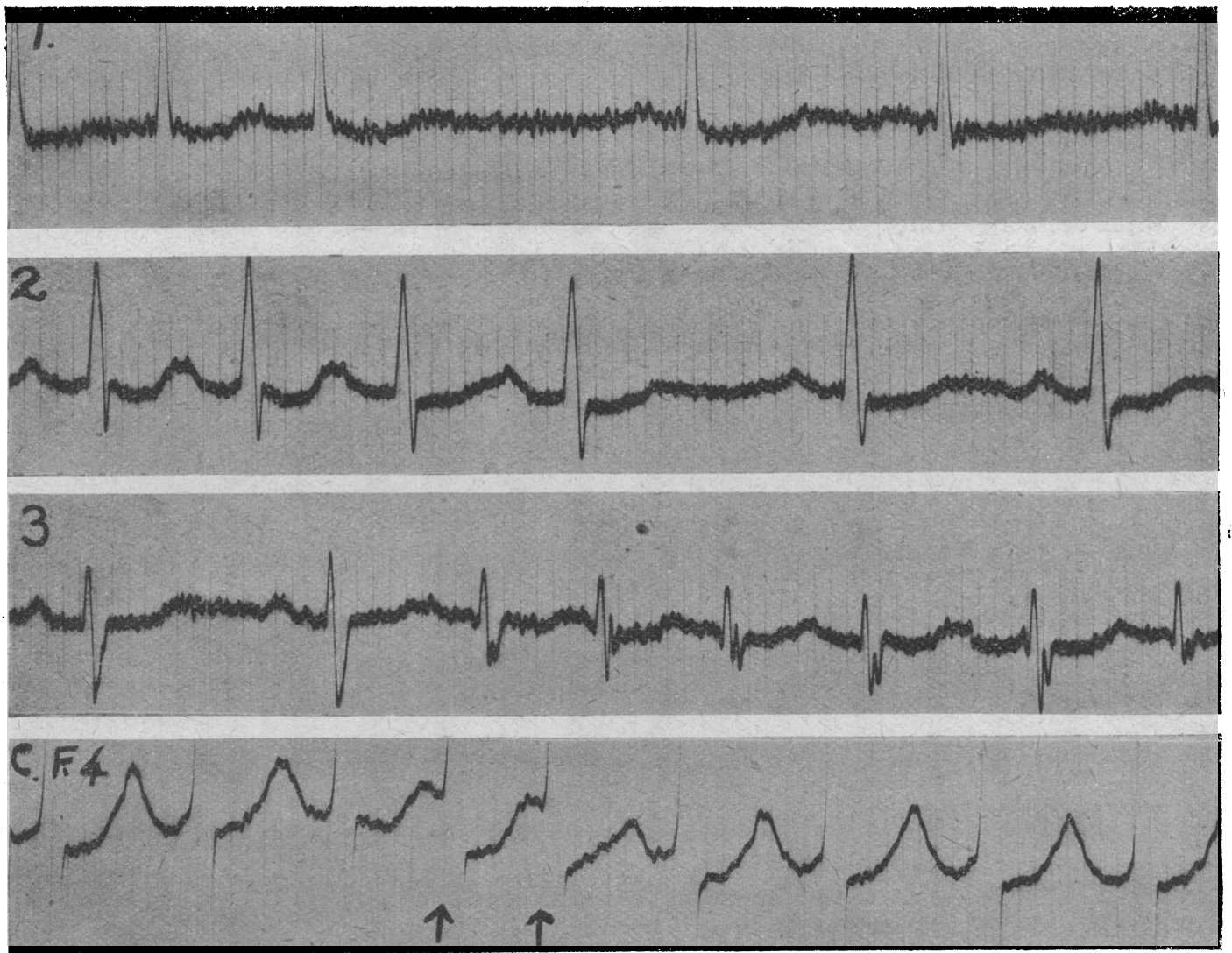

FIG. 3. - Case 3. Lead I shows the end of a period of rapid beating with the return of sinus rhythm, lead II shows an end of rapid beating and lead III the beginning of rapid beating with polymorphic ventricular complexes of supraventricular origin. The irregular arrhythmia has been called paroxysmal auricular fibrillation. CF4 shows two interruptions by $R$ waves of the antecedent $T$ waves. Time intervals $0.05 \mathrm{sec}$.

D 
deflection of a complex is interrupted by the initial sharp downward deflection of the next complex.

Case 3, male, aged 56 years. Myocardial degeneration and thyrotoxicosis. Paroxysmal supraventricular tachycardia probably auricular fibrillation. Admitted with breathlessness on exertion, of 6 months' duration. Thyroidectomy was followed 'by improvement in the physical condition. The basic rhythm is regular and of sinus origin. At times there is an irregular tachycardia with ventricular complexes which do not differ greatly from these found when the rhythm is regular (Fig. 3). The irregular rhythm is associated with variations in the shape of the ventricular complexes which, however, are supraventricular in origin. It is not possible entirely to eliminate multiple premature auricular systoles as a cause for the tachycardia but auricular fibrillation seems more likely.

Case 4, male, aged 71 years. Myocardial degeneration, auricular fibrillation, multiple premature ventricular systoles. Admitted to hospital because of a confused mental state. He was reasonably active for his years. Irregular rhythm due to auricular fibrillation and short runs of paroxysmal ventricular tachycardia. Interruptions of $T$ waves by $R$ waves occur frequently. Some of these are interruptions of ventricular complexes of supraventricular origin by premature ventricular complexes, others are of premature ventricular complexes by other premature ventricular complexes (Fig. 4A). Instances were observed of the interruption of a premature ventricular complex by a ventricular complex of supraventricular origin (Fig. 4 A and B) and of a ventricular complex of supraventricular origin by another ventricular complex of supraventricular origin.

Case 5, male, aged 71 years. Myocardial degeneration, auricular bigeminy, sinus bigeminy, multiple premature ventricular systoles. Admitted because of a right-sided inguinal hernia and found to have cardiac irregularity. In the last four months before admission there had been shortness of breath on exertion but no swelling of the ankles. (See Fig. 5.)

Case 6, male, aged 58 years. Cardiac infarction. Electrocardiograms showed a cardiac infarction and multiple premature ventricular systoles with interruptions of $T$ waves by $R$ waves (Fig. 6).

Case 7, female, aged 40 years. Myocardial damage of unknown origin, multiple premature systoles. Numerous examples of .ventricular premature systoles interrupting beats of normal sinus origin
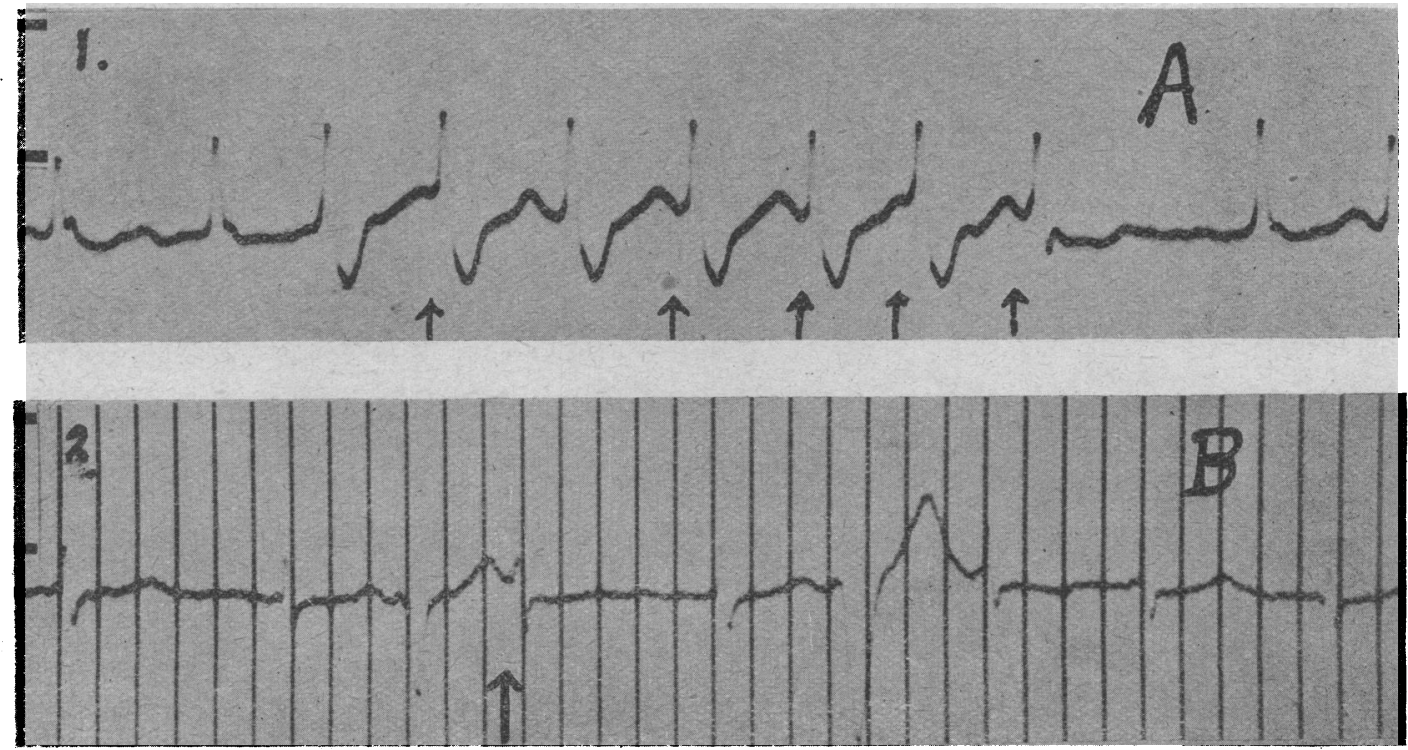

Fig. 4.-Case 4.

(A) Auricular fibrillation. The trace shows a run of six premature ventricular systoles followed by what appears to be a ventricular complex of supraventricular origin interrupting the $T$ wave of the last premature ventricular'systole.

(B) Auricular fibrillation. The trace shows the $\mathbf{T}$ wave of a premature ventricular complex interrupted by a ventricular complex which is clearly of supraventricular origin. Time intervals $0 \cdot 1 \mathrm{sec}$. 


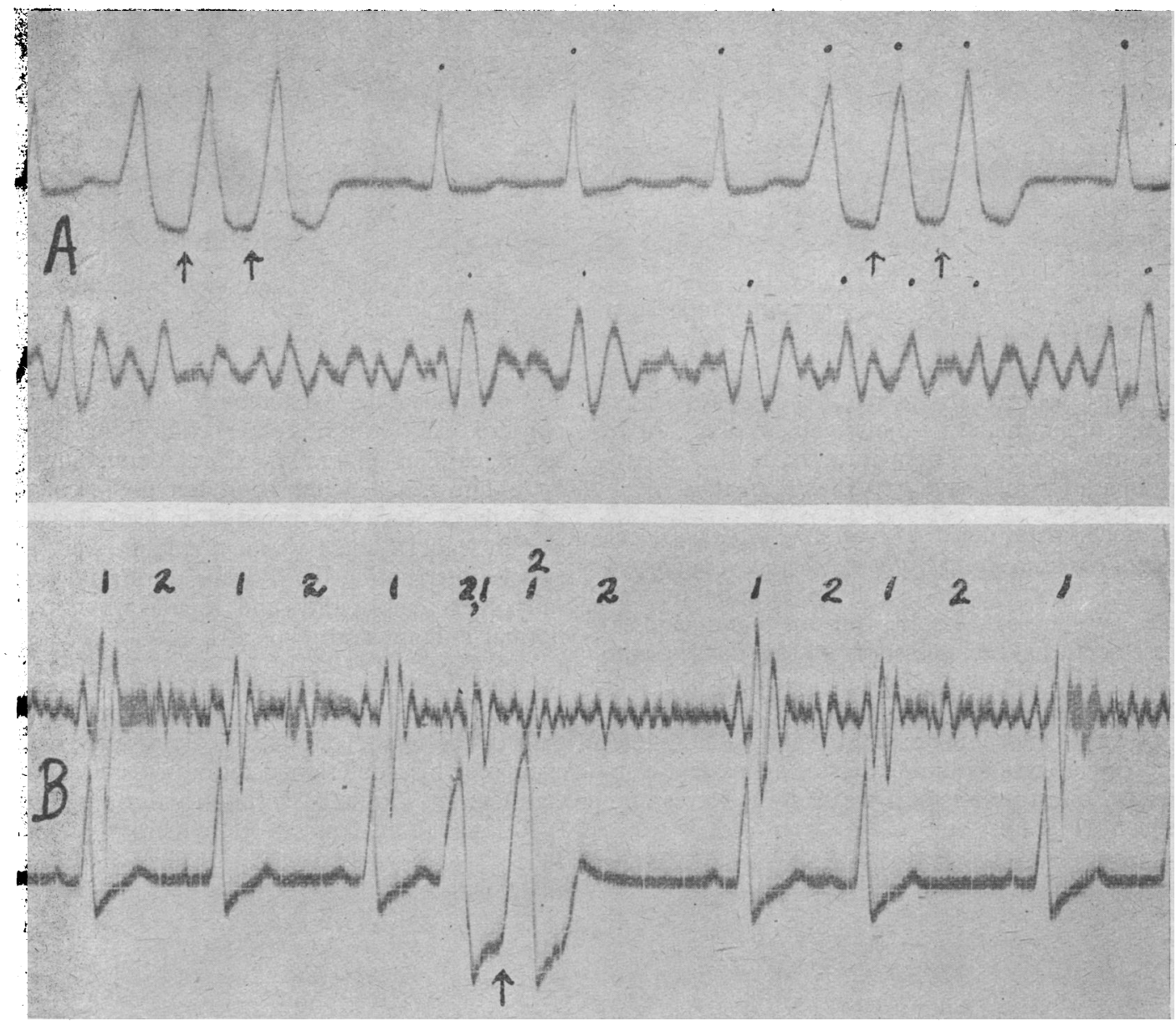

Fig. 5.-Case 5.

(A) The upper trace is an electrocardiogram recorded by a Matthews' oscillograph and shows auricular bigeminy broken by premature ventricular systoles which occur in two groups, each of three ventricular complexes. The 1 st and 2 nd premature ventricular systoles in each group are interrupted respectively by the 2 nd and 3 rd premature ventricular systoles. The shape of the uninterrupted 3rd systoles shows that the 1 st and 2 nd systoles are incomplete. The ballistocardiographic trace, using Malcolm's instrument, indicates a much smaller output of the heart from the premature than from natural systoles. Time trace $0.2 \mathrm{sec}$.

(B) The lower trace is a heart sound record and the lower trace. a simultaneous lead II electro-t cardiagram, both taken using a pair of Matthews' oscillographs. The latter shows a supraventricular bigeminy with wandering pacemaker and two premature ventricular systoles of which the second interrupts the first. The heart sounds alternate in intensity during the bigeminy, the louder sound following the longer diastole. The premature ventricular systoles give rise to weaker heart sounds. The second premature systole leads to a second heart sound. This raises the question of whether some filling of the heart took place between the two premature ventricular beats. Time trace $0.2 \mathrm{sec}$.

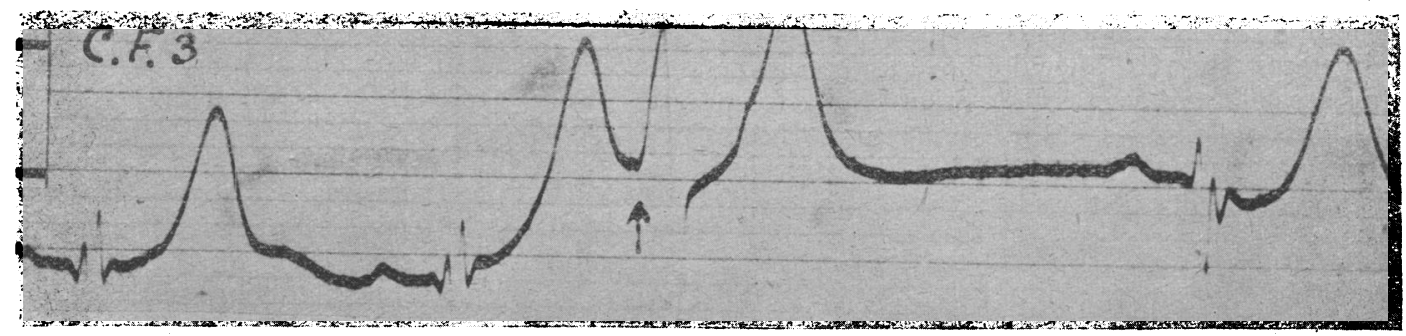

FIG. 6.-Case 6. The trace shows the interruption of a complex of sinus origin by a ventricular premature systole. at the point marked with an arrow. Time intervals 0.05 sec. 


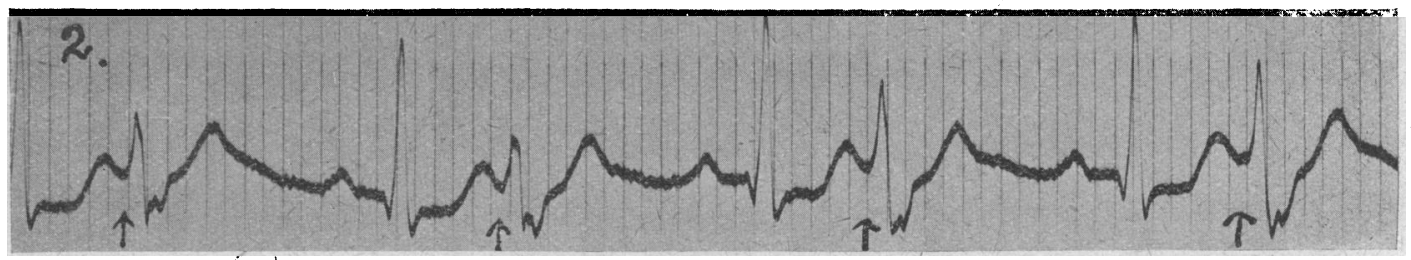

Fig. 7.-Case 7. The trace shows interruptions of the $T$ waves of complexes of sinus origin by the $R$ waves of premature ventricular systoles. Time intervals $0 \cdot 1 \mathrm{sec}$.

(Fig. 7). Examples were also encountered of polymorphic ventricular premature systoles interrupting other premature ventricular systoles. Apart from the numerous premature beats the electrocardiogram would have passed as normal.

Case 8, male, aged 77 years. Cardiac asthma, general congestive heart failure, benign arterial hypertension. Shortness of breath on exertion for two years, swelling of the ankles for one month, and extreme nocturnal breathlessness for four days prior to admission. The blood pressure was 220/108. The basic rhythm, of sinus origin, was interrupted by frequent polymorphic premature ventricular systoles occurring at various times after the antecedent complexes. Interruptions of $\mathrm{T}$ waves by $\mathrm{R}$ waves were frequent (Fig. 8). attack of pain which woke him in the early morning and continued for some hours in the absence of physical exertion; no other clinical evidence of recent cardiac infarction. Cardiograms suggested an old myocardial infarction but no recent damage: the $T$ wave was inverted in leads $I$ and IV, and in lead III there was a premature ventricular systole interrupting the $T$ wave during its descent (no other abnormality and regular rhythm with normal $\mathbf{P}-\mathrm{R}$ interval).

Case 11, male, aged 79 years. Recent cardiac infarction. This patient began with vomiting, and pain in the chest. He was obviously very ill and cyanosed, his condition deteriorated and he died. Cardiograms show a basic sinus rhythm with normal $\mathbf{P}-\mathbf{R}$ interval interrupted by numerous premature

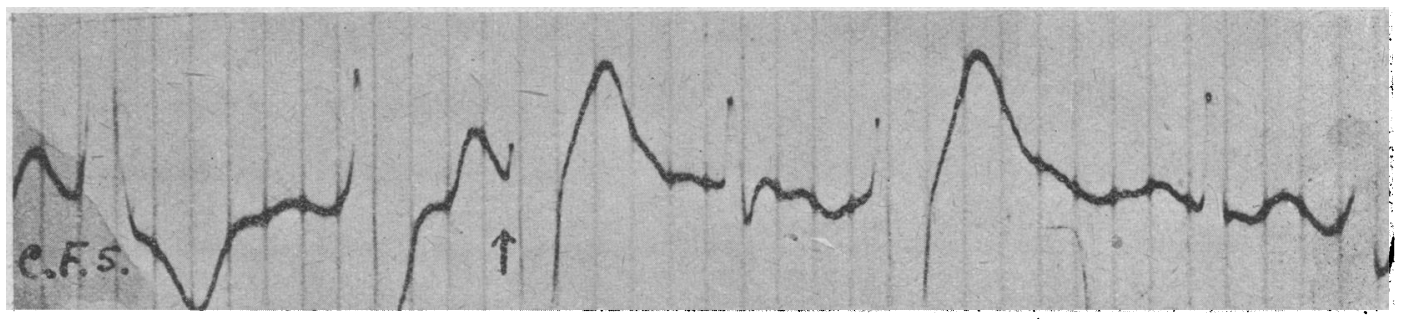

FIG. 8. - Case 8. The trace shows interruption of the terminal deflection of an ectopic ventricular complex by the initial deflection of another ectopic ventricular complex. Time intervals $0 \cdot 1 \mathrm{sec}$.

Case 9, male, aged 63 years. Posterior basal infarct. One month after the onset of the infarction, the patient, apparently well on the way to recovery, was telling the ward sister how well he felt and suddenly dropped back dead, apparently from ventricular flutter or fibrillation. The autopsy revealed a posterior basal cardiac infarction. Cardiograms showed interruption of ventricular complexes of supraventricular origin by ectopic ventricular complexes. The premature beats occurred at varying intervals after the previous beats but were not polymorphic.

Case 10, male, aged 77 years. Angina of effort, old cardiac infarction. Admitted because of an ventricular systoles. The QRS-T complexes were approximately $0.1 \mathrm{sec}$. in duration. There was a $6 \mathrm{~mm}$. Q wave in lead III with a sharply inverted $T$ wave. A clear interruption of the $T$ wave of $a$ complex of sinus origin by an ectopic ventricular complex occurred in lead $I$.

Case 12, male, aged 77 years. Myocardial degeneration. Short of breath and unable to hurry on level ground for six years prior to admission. Signs of congestive heart failure- were minimal. Sinus rhythm, but premature ventricular systoles occurred at fairly frequent intervals and several quite distinct interruptions of complexes of supraventricular origin by ectopic ventricular complexes occur. 
Case 13, female, aged 78 years. Cardiac asthma, auricular fibrillation, hypostatic pneumonia. Admitted with severe breathlessness which was thought to be the result of cardiac asthma. In hospital she developed a hypostatic pneumonia and died. Auricular fibrillation: QRS-T complexes of supraventricular origin sometimes occurred sufficiently early to interrupt the antecedent $T$ wave of other supraventricular complexes about half-way down their descents.

Case 14, female, aged 70 years. Acute exacerbation of chronic bronchitis, congestive heart failure, auricular fibrillation. The blood pressure was $180 / 80$. The congestive failure was treated by digitalis, the bronchitis improved, and the patient was discharged in fair condition. Auricular fibrillation: in CF2 and CF4 distinct interruptions of complexes of supraventricular origin by other complexes of supraventricular origin occurred. There was great variation in the shape of the ventricular complexes.

Case 15, female, aged 73 years. . Auricular fibrillation. Auricular fibrillation and interference with conduction in the branch bundles. Complexes of supraventricular origin were interrupted on occasion by other complexes of supraventricular origin and at times by ectopic ventricular complexes.

Case 16, female, aged 66 years. Paroxysmal auricular fibrillation. In two instances $\mathbf{R}$ waves were present on the descent of the antecedent $T$ waves showed a basic sinus rhythm; the ectopic ventricular complexes were polymorphic but falling, in regard to shape, into two main types of which one type only gave rise to interruptions of the $T$ waves of sinus beats.

\section{Findings IN THESE CASES}

The principal features of the 17 cases recorded with $R$ waves interrupting $T$ waves are set out in Table $I$. Most patients were in the later years of life, ten being over 70 and seven between 40 and 69 . There was a preponderance of males. Thirteen exhibited premature ventricular systoles; in one of these, only a single premature ventricular systole was present in the short length of tracing available; in the remaining 12 two or more ventricular premature systoles occurred, in tracings taken with the same lead, and the premature beats were polymorphic in 11 cases (Fig. 7 and 8 ). Of these 12 cases, 11 showed premature beats starting at irregular intervals of time after the antecedent normal beats. In the remaining case there were only two premature ventricular complexes and a conclusion as to whether the time interval was constant or variable could not be reached. Of the 12 cases with more than one premature systole 8 showed examples of pairs of premature beats occurring together. Some of these showed groups of three together and 3 of the 8 showed runs of paroxysmal tachycardia of five or more complexes (Fig. 2). In two of the cases where no such pairing of the ventricular premature systoles was discovered a considerable length of tracing was

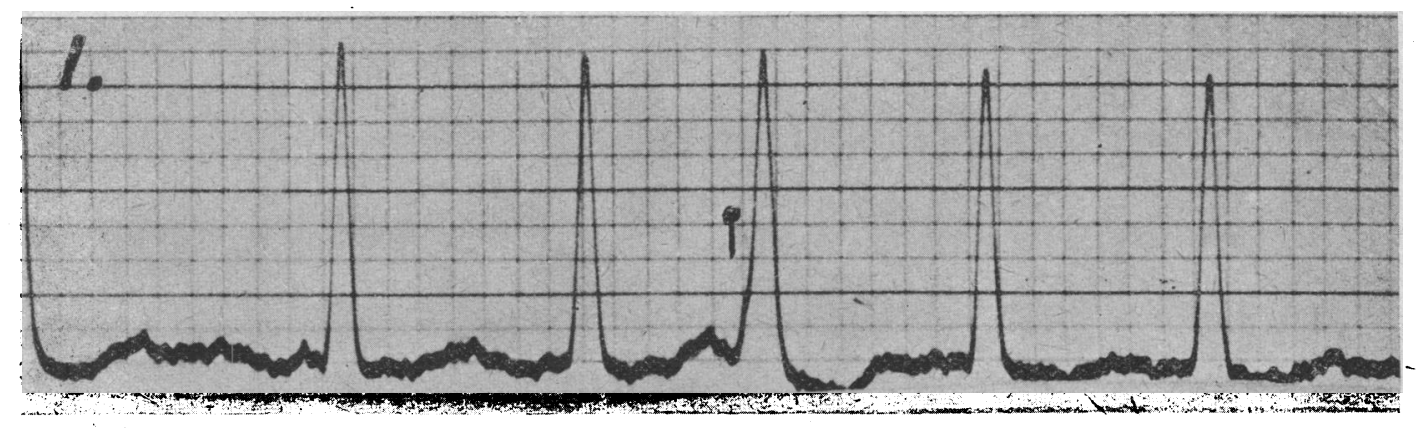

FIG. 9.-Case 16. Auricular fibrillation. T wave interruption. Time intervals $0.05 \mathrm{sec}$.

(Fig. 9). The QRS-T complexes showed variations in shape. There were no premature ventricular systoles.

Case 17, female, aged 62. Melancholia, multiple premature systoles. Admitted for electrical convulsive therapy on account of mental depression, hallucinations, and delusions. Electrocardiograms available and it seems likely that pairing of the beats was not occurring in these cases. In the other two cases only short lengths of tracing were available and pairing may or may not have been present at other times.

There were six cases with auricular fibrillation and it is interesting that all six showed polymorphic ventricular complexes of supraventricular origin 
(Fig. 3). This polymorphism is not usual in ordinary cases of auricular fibrillation and would appear to be a feature of cases in which $R$ waves appear upon $\mathrm{T}$ waves. Two of the six cases with auricular fibrillation also exhibited ectopic ventricular systoles and these also exhibited polymorphism. It was noted, but only when finally analysing the results, that three out of the six cases with auricular fibrillation (Fig. 3) sometimes showed sinus rhythm; this seems to be a higher proportion of paroxysmal auricular fibrillation than one would expect to encounter in a random collection of auricular fibrillation cases. From the above paragraphs it will be seen that in the 16 cases where it was possible to examine the phenomenon, polymorphism occurred in 15, being manifest either in ectopic ventricular complexes or in ventricular complexes of supraventricular origin or in both of these.

The four types of interruption that should occur theoretically have been found in the present series. Five patients were found in whom ventricular complexes of supraventricular origin interrupted other ventricular complexes of supraventricular origin and in one additional patient this probably occurred. These were also patients with auricular fibrillation and no such interruption occurred in any of the patients with sinus rhythm. Interruption of ventricular complexes of supraventricular origin by ectopic ventricular complexes (Fig. 6) and of ectopic ventricular complexes by other ectopic ventricular complexes (Fig. 1 and 4) both occurred fairly frequently, there being 9 patients who definitely, and three more who probably, exhibited the former phenomenon, and six patients who definitely exhibited the latter. Only one patient showed ectopic ventricular complexes being interrupted by ventricular complexes of supraventricular origin (Fig. 4) and this, as might be expected, was in a patient with both auricular fibrillation and multiple ectopic ventricular systoles.

One patient exhibited four types of interruption. Two patients exhibited two kinds of interruption and three patients probably had two types of interruption.

\section{EVIDENCE OF INTERRUPTION OF T WAVES BY R WAVES}

Some examples of the interruption of $T$ waves by $R$ waves have been presented. It may be considered that there has been an unmistakable interruption when a ventricular complex of well-defined shape has its tatter end cut off by the premature development of an $\mathbf{R}$ wave. In most cases complete ventricular complexes can be discovered which, up to the point of interruption, are identical with the interrupted complex, and a comparison of the com- plete and interrupted complexes makes it quite clear that there has been an interruption (Fig. 1). The distance between the $R$ wave of the interrupted complex and the $R$ wave of the interrupting complex, ordinarily, will be less than the length of the complete QRS-T complex. In patients who show the more striking interruptions of $T$ waves by $\mathbf{R}$ waves there are usually in addition a large number of interruptions which, however, are not so convincing - those where the $R$ wave arises low down on the descent of the $T$ wave, those where extracardiac potentials by deflecting the isoelectric base line are altering the shape of the complexes, and those where the interruption appears to be due to a prolongation of the interrupted $\mathrm{QRS}-\mathrm{T}$ complex as much as to prematurity of the $R$ wave.

\section{Other Features of Cases $R$ Waves on $T$ Waves}

Several of the examples of $R$ waves on $T$ waves have been encountered in cases of cardiac infarction especially about the end of the first week and the interruptions then are caused by premature ventricular systoles (Fig. 1 and 6).

In cases which are not cardiac infarctions a variety of clinical conditions have been associated with the appearance of $R$ waves on $T$ waves. Most of these patients show evidence of substantial myocardial damage. In addition, there is a similarity in the electrocardiographic features of all these cases which is not merely accidental.

(1) In 11 out of 12 cases, the ectopic ventricular complexes show variations in the shape (polymorphism). These variations in shape, however, are encountered not only when the premature ventricular systoles arise, as is usual in these patients, from multiple ectopic foci but also among ventricular complexes which arise in response to stimuli of supraventricular origin (Fig. 3).

(2) Ectopic ventricular beats often occur in pairs or triplets or in runs of ventricular paroxysmal tachycardia.

(3) The distances between premature beats and their antecedent normal complexes were variable. When pairs of ventricular premature systoles occur together, the distance between the two members of a pair is also a variable.

As will be seen later, the above changes may indicate increased excitahility of the myocardium.

The recognition of the fact that cases exhibiting interruptions of $T$ waves by $R$ waves commonly showed the above features, has led to a more careful, and often fruitful, search for interruptions, more particularly in cases where (a) ventricular complexes are polymorphic and at variable intervals after the antecedent beat, (b) the heart rate is fast and 
irregular, and (c) where some of the $\mathbf{R}$ waves arise close to the descending limb of the $T$ wave.*

\section{The Refractory Period of Cardiac Muscle}

The presence of $R$ waves on $T$ waves indicates that a new wave of excitation may arise within the heart before the previous wave of excitation has ended. This statement has reference to the heart
$\mathrm{U}$ wave. In the course of some observations with Mr. Fastier, direct leads from the surface of the heart have been taken in the dog in the course of the experimental production of ventricular flutter by the administration of amarin followed by adrenaline. In the stage preceding the onset of ventricular flutter we have observed interruption of the terminal deflection of these (direct lead) electrograms (Fig. 10)

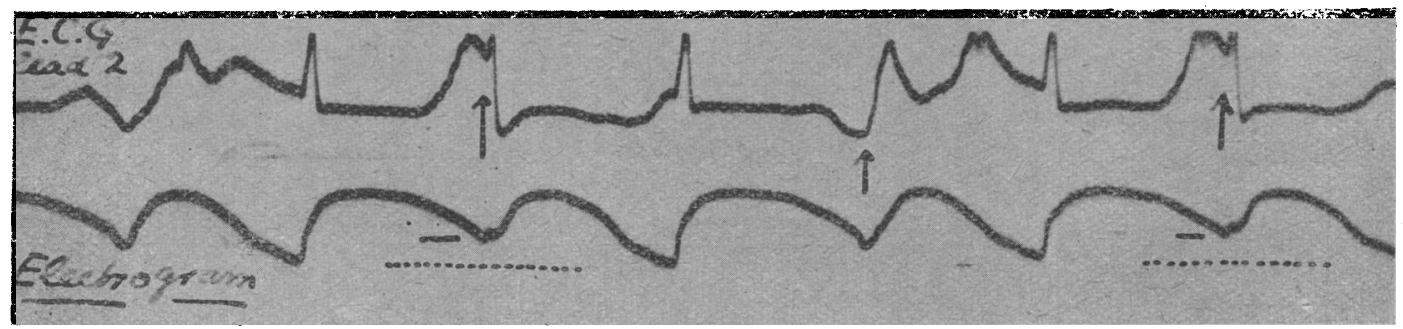

Fig. 10.-Dog (17 kg. 6.3 g.), under sodium barbitone. Vagi intact. The upper trace is an electrocardiogram which shows $R$ waves appearing on $T$ waves just before the onset of ventricular flutter, which was induced with adrenaline $(25 \mu \mathrm{g}$.) following treatment with amarin.

The lower trace is a simultaneously recorded electrogram, picked up from an electrode that was close to the A-V septum on the left side of the heart. This suggests practically continuous electrical activity in the ventricle at the stage of chaotic rhythm. Note that the electrogram does not always settle down'to a base line; where the $T$ wave of the electrocardiogram has been interrupted by the succeeding $R$ wave, the new electrogram may begin prematurely, as judged by the fact that the preceding complex does not fall to the level noted with the alternate series of complexes.

as a whole and in certain cases at least the termination of the $T$ wave does not represent the passing off of the wave of excitation throughout the entire heart. For example, in the case of a left ventricular premature systole, the right side of the heart is the last part to be excited and the terminal part of the $T$ wave represents the passing off of the wave of excitation from the right side. It is not so easy to explain the interruption of the $T$ waves of a supraventricular complex in this way for then, apart from branch bundle block, the $T$ wave represents the passing off of the wave of excitation on both sides of the heart. Such arguments do not go much further than indicating that we cannot eliminate the possibility that a single cardiac muscle fibre may be capable of responding to a new wave of excitation before the previous wave of excitation has ended.

The observations of Moe, Harris, and Wiggers (1942) indicate clearly that a suitable electrical stimulus applied to a ventricle during or just before the descent of the $T$ wave leads to a wave of excitation in the heart muscle, but after such a delay that the resulting $R$ wave came after the termination of the $T$ wave and commonly in the vicinity of the

* An additional 8 cases, including one with paroxysms of ventricular flutter (electrocardiographic), have been encountered while this paper was in press. by the succeeding wave of excitation, an observation that corresponds with the appearance of $R$ waves on $T$ waves but suggests that the phenomenon can take place even within the circumscribed area of the heart from which the electrogram is recorded. It is not unlikely therefore, that cardiac muscle fibres are capable, under certain circumstances, of responding to a new wave of excitation before the previous wave of excitation has ended.

\section{EXCITABILITY OF THE MYOCARDIUM AS THE UNDERLYING CAUSE}

The data concerning the 17 patients whose cases are reported suggest the probability that " increased excitability" of the myocardium may be an important underlying cause for the appearance of $R$ waves superimposed upon $T$ waves. It is necessary to consider further the meanings of the word excitability as applied to cardiac muscle. Increased excitability has been used in reference to an increased disposition of the cardiac muscle to originate new rhythms. More precisely, however, it should refer to the ease with which the myocardium responds when a stimulus is applied to it. No doubt, under clinical conditions, these two phenomena are difficult to differentiate from each other but evidence suggesting excitability in one sense or another is present in the great majority of cases here quoted. In Cases 1, 2, 


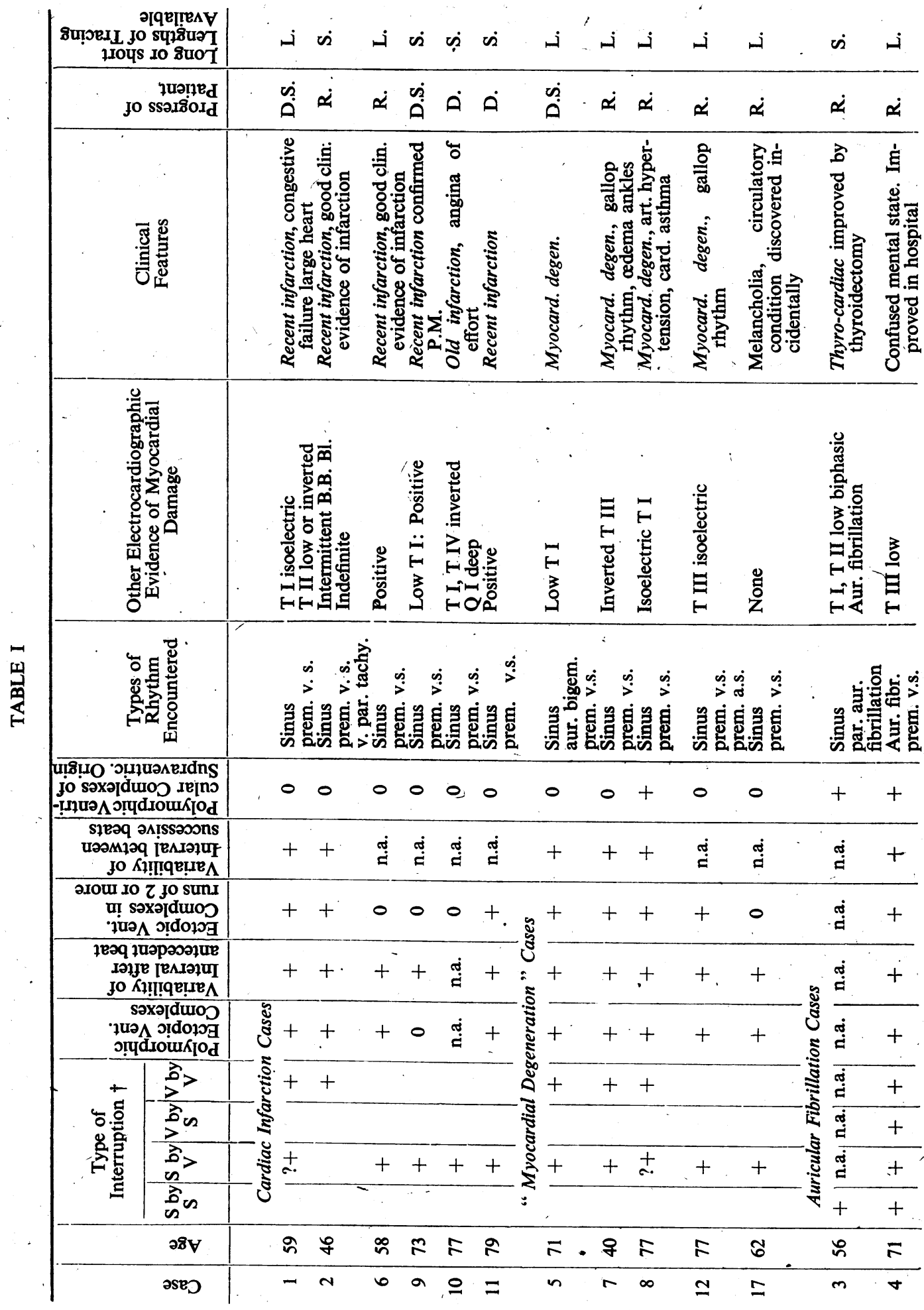



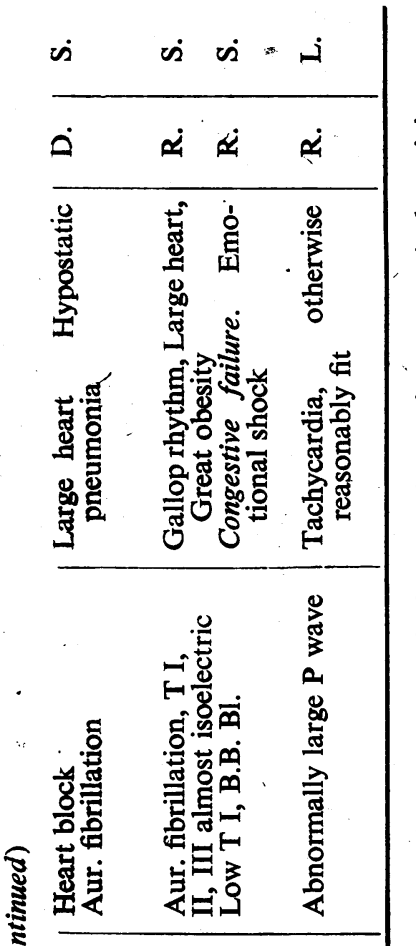

I

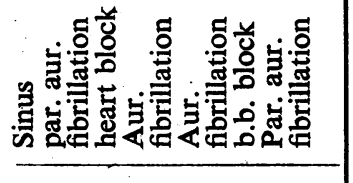

$+\quad++$

\begin{tabular}{|c|c|c|c|}
\hline 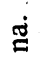 & 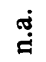 & 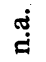 & 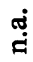 \\
\hline ه্] & ه્త & 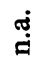 & 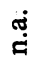 \\
\hline 迎 & $\underset{d}{\stackrel{d}{d}}$ & a. & 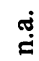 \\
\hline
\end{tabular}

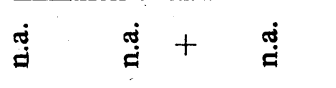

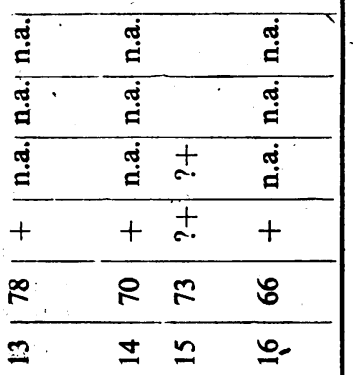

$4,5,7,8,9,12$, and 17 , it seems probable there is an increased tendency to originate new rhythms. Large numbers of premature ventricular systoles arise which, as judged from the shape of the complexes, originate from different foci. These ectopic beats occur at a variable time after the preceding beat and it may be thought from this that they probably result from the spontaneous activity of small parts of the cardiac muscle rather than from excitation of the ventricular muscle as the result of "re-entry" of impulses set up during the antecedent systole.

In contrast to this we have Cases $3,13,14$, and 16, with auricular fibrillation and an abnormally rapid beating of the ventricles. It may well be thought that the conducting tissue (less probably, ventricular muscle) is responding more readily than usual to the stimuli coming down to it from the auricles.

of the 17 cases, 6 are examples of cardiac infarction. In such cases the important pathological change in the myocardium is of comparatively recent origin. It is not surprising that in most cases where premature ventricular systoles are the result of a cardiac infarction the ectopic beats appears to arise from many foci. Likewise if these premature beats are arising spontaneously as the result of discharge from irritable foci, there is no obvious reason why they should develop at a fixed time after the antecedent normal complex and in fact, in none of the infarction cases here quoted, do we find the premature ventricular systoles at a fixed distance after the antecedent normal beat.

In the 6 cases of cardiac infarction described in this paper, $\mathbf{R}$ waves are found superimposed on $T$ waves, but in most cases of infarction this phenomenon is not found.

$R$ waves superimposed on $T$ waves were found in a miscellanerous group of cardiac cases where there was no cardiac infarction. In some of these patients ectopic beats arising in the ventricles are responsible for most of the interrupted complexes. These premature ventricular complexes arise from several foci and at variable times after the antecedent complexes: It seems probable that there is increased excitability of the ventricular muscle due in these cases to causes other than cardiac infarction. In other cases, however, the rhythm has a supraventricular origin. In most of such cases the evidence of excitability is two-fold, first the development of an irregular auricular tachycardia due in the present cases to auricular fibrillation and secondly the fact that the ventricles are able to respond to these stimuli by unusually rapid beating.

In some patients ectopic rhythms have arisen at one time from the auricles and at another from the ventricles. This observation also favours the idea 
that the common factor is an increase in myocardial excitability (Cases 4, 5, 12, and 15). Some further evidence is given in the succeeding section.

\section{The Supernormal Period of Cardiac EXCITABILITY}

As has been recognized for some time, there is a supernormal period in nerve at the time of the negative after-potential. Adrian (1921) showed that a super-normal period could be demonstrated in the ventricle of the frog upon acidifying the fluid in which the tissue was immersed. Wastl (1922) demonstrated supernormality in fatigued preparations. Cats anæsthetized with barbiturates invariably showed the supernormal period subsequent to the relative refractory period (Hoff and Nahum, 1938) but decerebrated cats rarely showed such super-normality (Eccles and Hoff, 1934). The super-normal period in the cat's ventricle may coincide with the terminal part of the $T$ wave and when a $U$ wave is present it falls during this stage. Wastl (1922) considers that the $U$ wave is in fact the terminal part of the ventricular complex.

It is interesting to consider whether any of the examples of $R$ waves upon $T$ waves are distributed in such a manner that the interrupting beats fall chiefly within a phase of super-normality. Supernormality might be concerned also in the frequency with which the ventricle is stimulated from auricles in a state of auricular fibrillation. If such were the case we would be dealing, presumably, with a supernormality of the conducting tissue. Supernormality of the conducting tissue has been postulated by Lewis and Master (1924) and by Goldenberg and Rothberger (1936). Super-normality might be exhibited in relation to premature ventricular systoles and might determine whether a stimulus arising from an irritable focus would be effective and lead to a premature ventricular complex during the supernormal phase of the antecedent heart beat.

In the cases described in this paper, most premature beats develop either in the $U$ wave region or on the descent of the $T$ wave. In some cases all the premature systoles are confined to these parts of the cardiac cycle. An example of this is found in Case 7. At times when this patient was not displaying $R$ waves on $T$ waves the premature beats occurred a little later in the cardiac cycle and mostly in the region of the $U$ wave. In some other patients (Case 8) the premature beats could occur during a greater part of the cardiac cycle but were concentrated particularly in the region of the $U$ wave or on the descent of the $T$ wave. Similarly, when premature beats interrupted other premature beats or were followed by other premature beats, the
$R$ wave of the second beat occurred very soon after the end of the $T$ wave or during its descent. The incidence of the premature beats in these patients could be explained as being largely determined by a super-normal period both in the case of premature beats following normal beats and in premature beats following other premature beats. In most patients where interruptions of premature beats by premature beats is observed, examples of the interruptions of normal beats by premature beats are also observed. The interruption is not so much determined by the nature of the complexes concerned as by a state of the cardiac muscle. Probably, the state of the cardiac muscle that allows the effective generation of these very premature ectopic beats is a state of super-normal excitability of the myocardium. It has been stated by Katz (1946) that a super-normal phase does not occur in the normal human heart but may be present in abnormal hearts. Scherf and Scholt (1939) also describe a super-normal phase in man. The observations reported in this paper are consistent with this view. The super-normal phase has been demonstrated in experiments on healthy cats but it has not yet been made clear whether under experimental conditions the heart was exhibiting a phenomenon that would not be obtained under more normal conditions.

\section{Relationships of Electrical and Mechanical EVENTS WHEN T WAVES ARE INTERRUPTED BY R WAVES}

It is not certain that the relationship between electrical and mechanical events in the heart is constant (Katz, 1946) and it may be changed both during health and in disease. In a number of our cases in which $R$ waves were encountered on $T$ waves phonocardiograms or alternatively ballistocardiograms were recorded simultaneously with the electrocardiograms. In Cases 5 (Fig. 5A, B) and 7 it was found that, where the $T$ wave was interrupted by the $\mathbf{R}$ wave of the premature systole, the intensity of the first sound produced by the very premature systole was less than that produced by normal heart contractions or by premature contractions that occur later in the cardiac cycle. Sometimes the weak first sound was followed by a second sound, in other cases no recorded second sound was observed. In those cases where a second sound was associated with a very early premature systole it is realized, with some surprise, that the premature beat must have caused a discharge of blood from the heart and the opening of the semilunar valves must have been sufficient to produce a second sound on closing again. Either the antecedent beat had not emptied the ventricles completely or some degree 
of filling of the ventricles must have taken place at a time corresponding to the lower third of the descent of the $\mathbf{T}$ wave. Other phonocardiograms gave results that were not dissimilar. In Case 5 several ballistocardiograms were taken (Fig. 5) and these showed, as expected, that the output from the heart was much less when a premature systole occurred very early in the cardiac cycle, but in all traces taken the ballistocardiographic results suggested that some blood had been discharged into the aorta even during the very premature systoles.

The Clinical Significance of R Waves on $T$ WAVES AND INDICATIONS FOR TREATMENT

A survey of published electrocardiograms shows that' $R$ waves on $T$ waves are often seen shortly before the onset of clinical ventricular flutter, also before the onset of experimental ventricular flutter produced in a variety of ways, and in this series $R$ waves on $T$ waves occurred in 6 patients with cardiac infarction of whom 2 died suddenly. These points indicate the possibility that the appearance of $R$ waves on $T$ waves may be of bad prognostic import. It would appear that the condition occurs chiefly among cases where there is good evidence of increased cardiac excitability and of substantial myocardial damage. Many of the patients were elderly. Naturally enough where there is already evidence of myocardial disease one may expect a poor prognosis; the question we must ask is whether the finding of an $R$ wave on a $T$ wave, in itself worsens the prognosis in these cases. On this question there is insufficient evidence but nevertheless the distinct indication that the subject deserves further study, first because of the known association, under experimental conditions, between the presence of $R$ waves on $T$ waves and ventricular flutter and fibrillation, secondly because in the clinical material studied there had been instances of sudden death that could be explained reasonably as examples of ventricular flutter or fibrillation. The matter is not without practical importance since preliminary observations suggest that the tendency to this particular manifestation can be arrested or greatly diminished by treatment with quinidine, digitalis, or strophanthus, more especially quinidine.

Unfortunately the possibility of a useful method of treatment did not emerge at the outset of this investigation, but in two cases of cardiac infarction exhibiting the presence of $R$ waves on $T$ waves the administration of quinidine arrested the premature systoles, and as premature ventricular systoles had been responsible for the interruptions of the antecedent complexes, this phenomenon was thereby eliminated. In the first (Case 6) the patient made an uninterrupted recovery from the infarction but was readmitted some, 3 months later with a second and fatal infarction. On this second occasion there were no premature systoles noted. In the second (Case 9) the administration of quinidine prevented the premature systoles and eliminated the interruption of complexes. Unfortunately a hæmatemesis necessitated withdrawal of the quinidine whereupon the premature ventricular complexes recurred. One month after the onset of the infarction the patient was saying how well he felt and he suddenly stopped and was dead in a few seconds. This would appear to be an example of ventricular flutter. In Case 7 the patient was in moderately good condition with little breathlessness and a moderate exercise tolerance, but a gallop rhythm. She was treated with quinidine; this stopped almost all ectopic beats, but unfortunately the drug had to be stopped as she experienced continuous vertigo and headache. On administering strophanthin by intravenous injection the number of premature systoles were much reduced, and those which occurred were found later in the cardiac cycle and after the termination of the $T$ wave. With eight tablets daily of $1 / 500$ grain of strophanthin by mouth; also, the premature systoles were much reduced in number. In view of the increased excitability of the ventricle brought about by substances such as digitalis and strophanthus, 1/200 of a grain of atropine was given thrice daily at the start; this to some extent at least, serves as an anti-fibrillating agent. The patient was much improved and was discharged from hospital.

It would seem that the administration of quinidine, digitalis and strophanthin in such cases deserves a trial. Perhaps quinidine is the most likely to prove satisfactory and digitalis and strophanthus are probably potentially dangerous. In any case where cardiac infarction has taken place neither digitalis nor sţrophanthus should be given unless there is congestive heart failure.

\section{SUMMARY}

Seventeen cases are described in which $R$ waves appear on the descent of $T$ waves. They appear to be examples of an electrocardiographic syndrome characterized by a group of related abnormalities some of which are explicable in terms of increased myocardial excitability.

The presence of an $R$ wave on a $T$ wave indicates that a new wave of excitation starts before the previous wave has ended. It is convenient in these circumstances to speak of a ventricular complex being interrupted before its completion.

Ventricular complexes of supraventricular origin may be interrupted by other ventricular complexes 
of supraventricular origin or by ectopic ventricular complexes. Ectopic ventricular complexes may be interrupted by ventricular complexes of supraventricular origin or by other ectopic ventricular complexes.

$\mathbf{R}$ waves on $\mathbf{T}$ waves may occur with ectopic ventricular systoles, paroxysmal ventricular tachycardia, auricular fibrillation, and auricular paroxysmal tachycardia. They may occur in cardiac infarction. Theoretically they might occur with ectopic auricular systoles and auricular flutter.

When $R$ waves on $T$ waves are due to ectopic ventricular complexes, these are almost always polymorphic and presumably multifocal. The ectopic ventricular complexes occur at varying intervals after the antecedent beat and there is a tendency for them to occur in runs of two or three or more beats. Even when the presence of $R$ waves is brought about by a supraventricular rhythm (usually auricular fibrillation), polymorphic ventricular complexes ate often found.

In view of the fact that $R$ waves on $T$ waves are frequent precursors of clinical and experimental ventricular flutter and ventricular fibrillation, it is desirable to consider the significance of this finding for prognosis in man. A few of the patients in this series have died suddenly.

It is probably advantageous to use quinidine in such patients with multiple premature ventricular systoles. This abolished the interruption of $T$ waves by $\mathbf{R}$ waves in all of five cases. Digitalis and strophanthus also abolish the interruptions in some cases, but have theoretical objections.

The significance of this phenomenon in relation to the refractory period of heart muscle, the supernormal phase of cardiac excitability, and the mechanical changes associated with very premature systoles are discussed.

I am indebted to Dr. Laurence Malcolm for permission to use his ballistocardiograph, to Dr. Iverach, Dr. Kirk, and Dr. Harold Palmer for permission to examine cases under their care, and to the New Zealand Medical Research Council for the expenses of the research.

\section{REFERENCES}

Adrian, E. D. (1921). J. Physiol., 55, 193.

Ashman, R., and Hull, E. (1941). Essentials of Electrocardiography, The Macmillan Company, New York, p. 181.

Eccles, J. C., and Hoff, H. E. (1934). Proc. Roy. Soc., 115B, 307.

Fastier, F. N., and Smirk, F. H. (1948). J. Physiol., 107, 318.

Goldenberg, M., and Rothberger, C. J. (1936). Pfluger's Arch., 237, 295.

Graybiel, A., and White, P. D. (1946). Electrocardiography in Practice, W. B. Saunders Company, Philadelphia and London, p. 81 .

Hoff, H. E., and Nahum, L. H. (1938). Amer. J. Physiol., 124, 591 .
Katz, L. N. (1928). Physiol. Rev., 8, 447.

(1946). Electrocardiography, Lea and Febiger, Philadelphia, p. 71.

Lewis, T., and Master, A. M. (1924). Heart, 11, 371.

Moe, G. K., Harris, A. S., and Wiggers, C. J. (1942). Amer. J. Physiol., 134, 473.

Scherf, D., and Boyd, L. J. (1940). Clinical Electrocardiography, William Heinemann, Ltd., London, 181.

and Scholt, A. (1939). Amer. Heart J., 17, 357.

Wastl, H. (1922). Zitschr. Biol., 75, 289. Cited by Hoff and Nahum (1938).

Wenckebach, K. F., and Winterberg, H. (1927). Cited by Katz (1928). 